livraisons

d'Histoire

de l'Architecture

\section{Livraisons de l'histoire de l'architecture}

41 | 2021

L'architecte en son agence

\title{
Quelques éléments de réflexion sur l'élaboration collective des projets architecturaux médiévaux
}

Some elements of reflection on the collective elaboration of medieval projects Gedanken zur gemeinschaftlichen Erarbeitung mittelalterlicher

Architekturprojekte

\section{Philippe Bernardi}

\section{(2) OpenEdition}

Journals

Édition électronique

URL : https://journals.openedition.org/lha/3004

DOI : 10.4000/lha.3004

ISSN : 1960-5994

Éditeur

Association Livraisons d'histoire de l'architecture - LHA

Référence électronique

Philippe Bernardi, «Quelques éléments de réflexion sur l'élaboration collective des projets architecturaux médiévaux », Livraisons de l'histoire de l'architecture [En ligne], 41 | 2021, mis en ligne le 15 juin 2021, consulté le 19 juin 2021. URL : http://journals.openedition.org//ha/3004 ; DOI : https:// doi.org/10.4000/lha.3004

Ce document a été généré automatiquement le 19 juin 2021.

Tous droits réservés à l'Association LHA 


\title{
Quelques éléments de réflexion sur l'élaboration collective des projets architecturaux médiévaux
}

\author{
Some elements of reflection on the collective elaboration of medieval projects \\ Gedanken zur gemeinschaftlichen Erarbeitung mittelalterlicher \\ Architekturprojekte
}

Philippe Bernardi

1 L'anachronisme que peut revêtir, a priori, l'emploi de l'appellation «agence d'architecture » pour le Moyen Âge n'est pas sans rappeler celui attaché à l'usage même du terme « architecte » pour cette période. L'emploi des mots architectus ou architector demeure, en effet, relativement confus dans les textes médiévaux ${ }^{1}$. Et il est clair que la figure moderne de l'architecte, qui dresse le plan, établit le devis, dirige les travaux, vérifie et règle les mémoires de l'entrepreneur, n'émerge que très progressivement et imparfaitement à partir du XIII ${ }^{e}$ siècle. Sans précisions sémantiques préalables, le recours au terme reviendrait à nier toute la dimension historique de la figure de l'architecte comme la spécificité de celle du " maître d'œuvre » (magister operis) voire de l'architectus médiéval. Il est entendu que la notion contemporaine d'architecte ne saurait s'appliquer stricto sensu à la période médiévale, elle a néanmoins pu et peut encore servir à apprécier les principes de fonctionnement propres aux chantiers de cette époque ancienne. Pourquoi n'en serait-il pas de même de l'idée d'agence d'architecture? Définie comme "organisme dans lequel des architectes, ingénieurs, dessinateurs et représentants d'autres disciplines élaborent des projets architecturaux pour le compte de l'État, d'une collectivité territoriale ou du secteur privé $»^{2}$, l'agence d'architecture a le mérite d'interroger l'historien sur le caractère collectif de l'élaboration des projets. C'est sous cet angle que la présente contribution se propose d'aborder ce thème de "L'architecte en son agence». Pour ce faire, il conviendra en premier lieu de revenir sur l'image de créateur attachée à l'architecte ou au maître d'œuvre médiéval telle qu'elle s'impose dans l'historiographie. Nous porterons ensuite notre attention sur le moment particulier de l'élaboration des projets, à partir de la 
documentation conservée pour quelques grands chantiers occidentaux de la fin du Moyen Âge, afin de mettre en évidence les diverses collaborations auxquelles cette phase de la construction pouvait donner lieu. Nous tenterons, enfin, de voir si ces collaborations pouvaient avoir une forme relativement pérenne et ébaucher les contours d'une proto agence d'architecte.

\section{L'architecte comme auteur ${ }^{3}$}

2 L'architecte ou le maître d'œuvre, peu importe le qualificatif choisi, est un personnage central des travaux consacrés à la construction médiévale. Son identification est une question récurrente dont témoignent en creux les études portant sur l'anonymat des grands édifices gothiques puisqu'il est souvent envisagé comme la marque d'une absence d'individualisation du concepteur au sein de l'équipe de bâtisseurs ${ }^{4}$. Or cette absence d'individualisation ne remet pas en cause l'existence d'un concepteur unique dont la figure émergerait progressivement de la masse indistincte des constructeurs à partir du XIII siècle. Avec la relative valorisation que connaissent les activités matérielles (ou arts mécaniques) à partir des XII ${ }^{e}$-XIII ${ }^{e}$ siècles, s'ouvrirait alors le chemin sinueux qui «mène l'architecte à la reconnaissance de son activité en tant qu'art libéral $»^{5}$. L'une des mutations les plus importantes que connaît alors le métier d'architecte consiste dans l'autorité acquise par la maitrise du dessin qui lui permet de transmettre ses instructions à des exécutants sans être tenu de suivre en permanence le chantier. Lente, cette transformation ne trouverait toutefois son achèvement qu'au $\mathrm{XVI}^{\mathrm{e}}$ siècle avec des figures comme celle de l'architecte Galeazzo Alessi ${ }^{6}$. Cette évolution, sur laquelle il n'est pas question ici de revenir plus en détail, incline à voir dans l'architecte un artiste concevant des bâtiments plus qu'un technicien. La figure de l'architecte en tant qu'artiste et auteur se présente ainsi comme le fruit d'une mutation conduite sur plusieurs siècles, mais c'est pourtant elle qui est convoquée quand il s'agit d'envisager la conception d'un édifice gothique, suivant ce que Franco Borsi désigne à juste titre comme «notre habitude invétérée à considérer l'histoire par auteurs et par textes, c'est-à-dire par architectes et par architectures suivant un modèle standard anhistorique et antihistorique du rapport auteur-œuvre $»^{7}$. Cette vision parait étayée par la valorisation sociale de ce que l'on a coutume de présenter comme « de grandes figures d'architectes». C'est un Jean de Chelles, connu par une inscription sur le bras nord du transept de Notre-Dame de Paris qui le présente comme magister cementarius (maître maçon), ou un Pierre de Montreuil, qui lui succéda à Notre-Dame et que son épitaphe, célèbre, qualifie de vivens doctor lathomorum (de son vivant, docteur [dans l'art] des tailleurs de pierre), par exemple ${ }^{8}$. L'œuvre ne saurait être, semble-t-il, que de conception individuelle et la plupart des travaux savants consacrés aux chantiers des cathédrales gothiques comme à la construction au Moyen Âge (les deux ne faisant souvent qu'un jusque dans les années 1970 en France), font la part belle à "l'architecte", entendu avant tout comme un concepteur ou plutôt comme le concepteur du monument ${ }^{9}$. L'évolution historique de leur position ne porterait, en quelque sorte, que sur leur participation active aux travaux en tant que cementarius ou lapicida.

3 Soulignons que nous disposons toutefois de peu de renseignements sur les attributions exactes de ces personnages au sein du chantier ou sur leur manière de travailler, $y$ compris pour le $\mathrm{XV}^{\mathrm{e}}$ siècle. Peut-on, en outre, affirmer que le nom inscrit, sur une 
plaque commémorative ou sur un contrat, ne désigne qu'un individu, ou bien faut-il imaginer que c'est un groupe, une familia composée de parents mais également d'associés ou d'employés de toutes sortes, qui intervient au nom de son chef?

\section{Le projet : œuvre individuelle ou collective?}

4 L'iconographie et les textes médiévaux, il faut bien le reconnaître, placent souvent à la tête du chantier la figure éminente d'un homme seul. C'est le cas, par exemple, dans une miniature figurant, au XIII ${ }^{e}$ siècle, la construction de la cathédrale de Modène (Ill. 1). Nous y observons, campé sur la gauche, Lanfranc, qualifié par ailleurs, nous le verrons, d'architector. Il tient à la main une sorte de bâton et semble diriger d'un geste de l'index le travail que doivent accomplir les hommes affairés qui lui font face. D'un côté, Lanfranc, et de l'autre, la troupe des hommes de l'art (artifices) et leurs manœuvres (operarii) dans ce que l'on peut légitimement considérer comme une division entre direction du chantier et travail de construction. Il est tentant de voir dans cette représentation une illustration du fameux sermon de Nicolas de Biard, qui lui est contemporain. Dans ce texte de 1261, le prédicateur dénonce l'attitude des maitres des maçons qui «baguette et gants à la main, disent à d'autres : «Par ici me le taille»; ils ne travaillent pas et reçoivent cependant un plus grand salaire $»^{10}$. Ce passage de sermon est souvent pris comme le témoignage d'une réaction face à une évolution qui verrait l'architecte laisser la réalisation à d'autres (« ils ne travaillent pas ») pour se consacrer à l'invention et au projet. Faut-il toutefois voir dans ces maîtres des maçons (magistri cementariorum) des concepteurs occupés à distribuer l'ouvrage ou bien des contremaîtres placés à la tête d'équipes de tailleurs de pierre ? Et Lanfranc, dans la miniature présentée, n'est-il pas simplement en position de direction du chantier? 


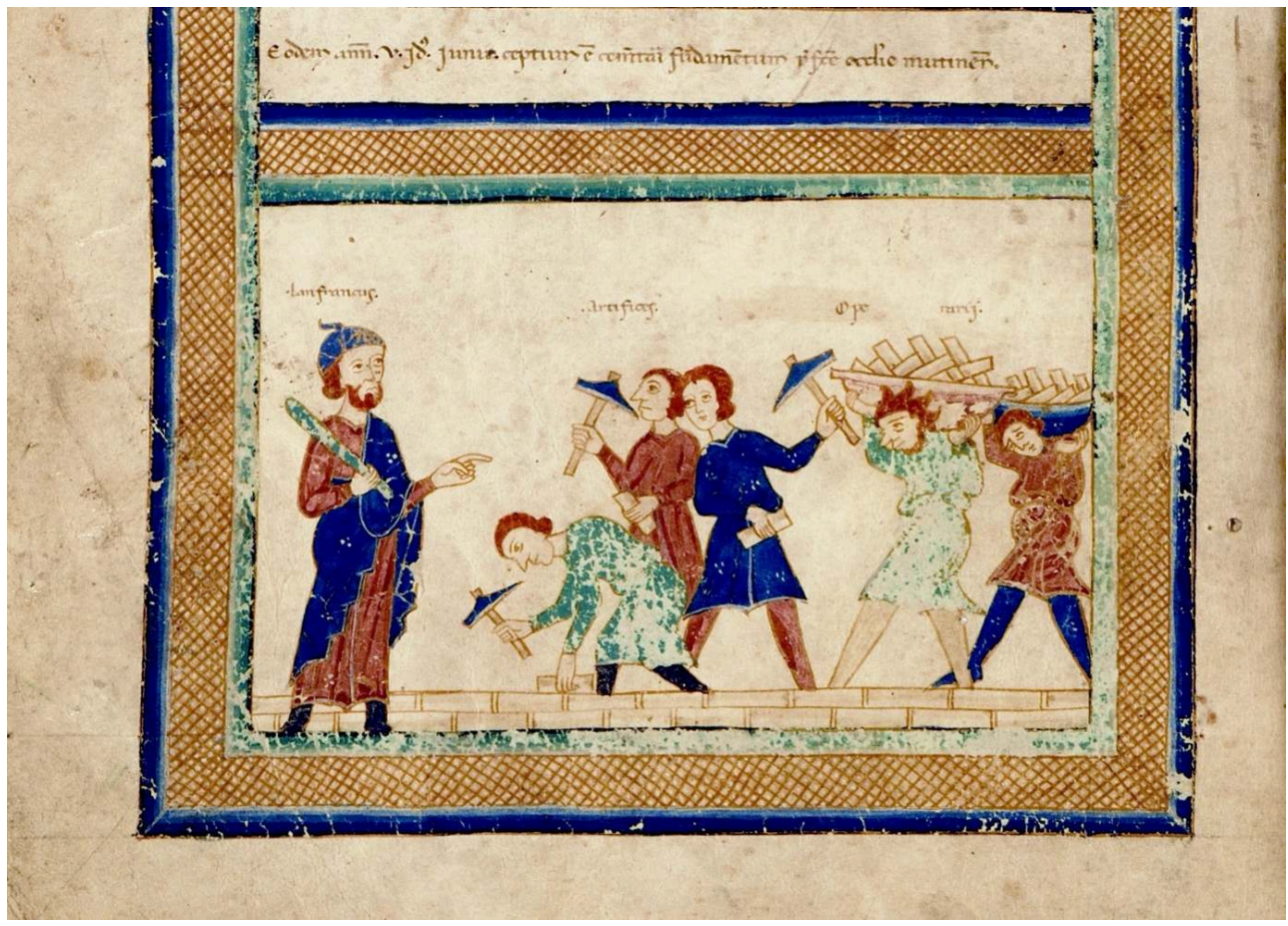

"La construction de la cathédrale de Modène », miniature du XIII siècle tirée de la Relatio de innovatione ecclesie sancti Geminiani mutinensis conservée dans la Bibliothèque capitulaire de Modène. Archivio capitolare del duomo di Modena, codex 0-II-11, fol 1v - photographie : G. Roncaglia. Extrait.

Notons que cette miniature prend place dans une page dont elle n'occupe que la partie basse (Ill. 2). L'image supérieure illustre une phase antérieure de la construction avec le creusement des fondations de la future église. Lanfranc y est désigné comme Lanfrancus architector. Il se présente face aux terrassiers (operarii), mais il n'est plus seul alors. Il précède légèrement un groupe de six hommes dont il se distingue par sa stature, sa tenue et son bâton. Qui sont ces personnages qui assistent au démarrage des travaux et à cette phase essentielle, intermédiaire entre l'élaboration du projet et la construction à proprement parler? Aucune inscription ne vient le préciser et leur tenue vestimentaire ne permet pas d'y voir des ecclésiastiques ou des membres de la noblesse qui interviendraient ici en tant que mécènes ou commanditaires. S'agit-il de la foule des fidèles ou de personnages au moyen desquels le peintre a simplement cherché à équilibrer la scène ? Il est malaisé, en l'absence d'autres éléments, de percer le mystère de cette présence mais relevons que c'est au milieu de ce groupe, précisément, que Lanfranc est qualifié d'architector. La mise en regard des deux scènes de la page pose question et interroge sur la solitude présumée de l'architecte médiéval. 


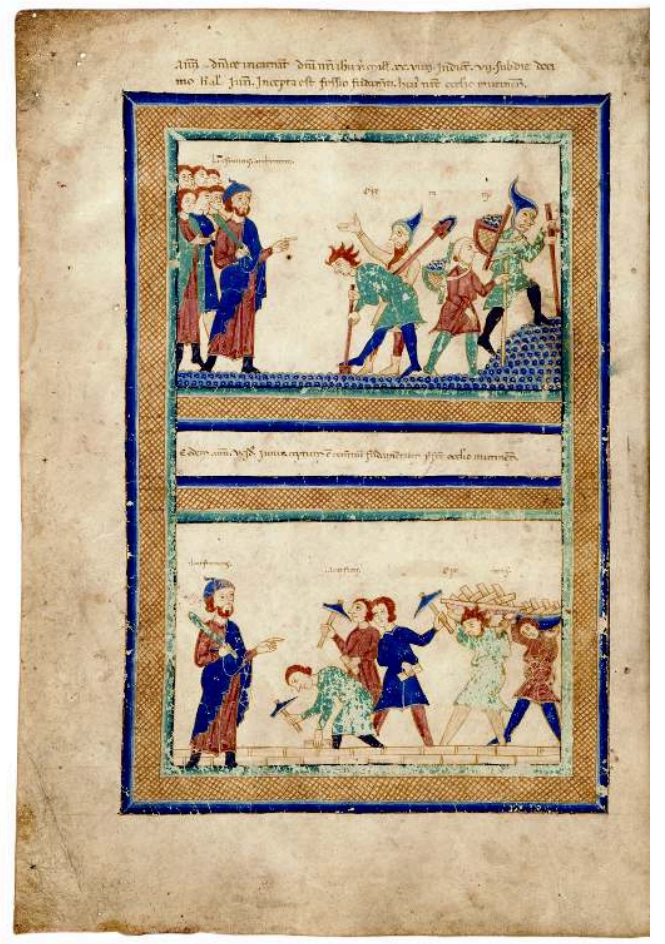

Miniature du XIII' siècle tirée de la Relatio de innovatione ecclesie sancti Geminiani mutinensis conservée dans la Bibliothèque capitulaire de Modène.

Archivio capitolare del duomo di Modena, codex 0-II-11, fol 1v - photographie : G. Roncaglia.

6 À en juger par les débats auxquels donna lieu l'élaboration de certains projets, cette phase du travail de conception ne semble pas avoir été strictement ou toujours solitaire. Les sources médiévales font en effet état de disputes fameuses ou de consultations engagées afin d'arrêter d'importants choix constructifs. C'est le cas à Salisbury, en 1174, à Sienne, en 1322, à Milan, à partir de 1391 ou à Gérone, en 1416, entre autres ${ }^{11}$. Ces débats sont souvent présentés comme des confrontations entre architectes, comme des affrontements entre divers projets mais ils ne semblent pas être que cela. Prenons le cas de Gérone. En 1416, se pose la question de rebâtir la nef romane pour l'accorder au chevet gothique qui avait déjà été élevé, mais devait-on le faire avec une nef unique ou avec trois nefs ? Le maitre d'œuvre Guillem Bofill, en charge depuis 1402 du chantier réunit alors, sur ordre de l'évêque, pas moins de douze maîtres venus de Tarragone, Tortosa, Barcelone, Urgell, Manresa, Castello d'Empurias, Perpignan et Narbonne. Si les intérêts des uns et des autres pouvaient diverger, il ne s'agissait pas, alors, de confronter les projets élaborés par ces spécialistes mais de répondre à une série de questions : Peut-on, en toute sécurité, donner à la cathédrale une nef unique? Si la nef unique est impossible, que pensez-vous du type à trois vaisseaux? En tout état de cause, quelle sera la forme de la nef la plus convenable et la mieux proportionnée au chevet déjà achevé ? Le propos, on le voit, est de recueillir des avis, d'échanger, de nourrir la réflexion et de mûrir le projet.

7 À Milan, en 1392, c'est à quatorze ingegneri que furent posées onze questions sur le projet élaboré par Heinrich Parler pour la cathédrale. Mais c'est la passe d'armes qui eut lieu en 1400-1401, entre l'architecte français Jean Mignot et les ingénieurs de la 
fabrique de cette église qui fournit à ce dernier l'occasion de lancer son «ars sine scientia nihil est » (l'art n'est rien sans la science), auquel ses adversaires répondirent que "scientia sine arte nihil est" (la science n'est rien sans l'art) ${ }^{12}$. Même tendu, l'échange demeure et la consultation joue son rôle de creuset d'où doit émerger le projet. Au-delà du caractère marquant de la formule employée et de la hiérarchie qu'elle cherche à établir, ce sont les conditions mêmes de l'échange qui sont intéressantes pour notre propos. Face à Jean Mignot, lui aussi qualifié d'ingegnere, ne se trouvent pas des concurrents venus chacun défendre leur projet mais un groupe d'hommes parlant d'une seule voix pour justifier les options qui avaient été retenues.

8 Les exemples de Gérone et de Milan témoignent de consultations et de réflexions collectives attestées beaucoup plus largement à travers la diffusion du recours aux expertises. Cette thématique a, récemment, fait l'objet de plusieurs publications collectives majeures qui ont permis de replacer l'expertise architecturale dans un mouvement plus ample et de mieux saisir les pratiques en vigueur à la fin du Moyen Âge ${ }^{13}$. L'expertise y apparaît relativement codifiée, confiée pour les périodes les plus anciennes à des hommes simplement reconnus pour leur droiture (boni ou legales homines - bons ou loyaux hommes) puis de plus en plus, à partir du XIII e siècle, à des hommes de l'art désignés comme magistri ou magistri in talibus experti (maîtres experts en cela) puis comme experti avant que leur fonction ne se professionnalise, en France, dans le courant du XVI siècle $^{14}$. Or ces experts interviennent généralement à plusieurs, visitent les lieux et rendent un rapport commun qui se propose comme une synthèse de leurs observations. C'est le rôle qui incombera souvent aux ingénieurs royaux anglais à partir du règne d'Henri II (1154-1189) et plus largement aux maîtres des œuvres royaux, comtaux ou autres dont les derniers siècles du Moyen Âge livrent de nombreux exemples ${ }^{15}$. Envisager le cas spécifique de ceux-ci dans le détail nous éloignerait toutefois un peu trop de la question du caractère collectif ou non de l'élaboration du projet. Contentons-nous de relever que le rôle des experts peut, dans bien des cas, se limiter à un contrôle a posteriori mais qu'il n'en va pas toujours ainsi. Ce peut être bien en amont de la construction, au moment où le projet prend forme, que le conseil est demandé. La chronique de Jacques de Guise raconte ainsi, vers 1225-1233, que, quand la comtesse Jeanne de Flandre envisagea l'installation d'un couvent de franciscains à Valenciennes, elle "envoya" un groupe d'experts (architectores et carpentarios « architectes » et charpentiers) pour reconnaître la stabilité du terrain choisi ${ }^{16}$.

9 La question des fondations apparaît particulièrement importante pour la viabilité du projet, ce que de nombreuses expertises commandées pour cette phase des travaux confirment à leur façon. En l'absence de données précises sur la nature du sous-sol, c'est le creusement des tranchées de fondation qui permet d'éprouver la résistance du terrain et d'apprécier la pertinence des solutions envisagées. L'intervention d'experts peut, en ce sens, trouver place dans le processus d'élaboration du projet architectural.

10 Ces quelques éléments épars ne permettent certes pas d'affirmer que le personnage éminent désigné comme "architecte " était systématiquement entouré d'une équipe. Ils laissent entendre, à tout le moins, que le conseil, la discussion et l'échange sur le projet en cours d'élaboration pouvaient exister et relevaient même de pratiques assez courantes pour que la documentation conservée en garde de nombreuses traces.

11 Le dessin lui-même était-il un exercice solitaire ? Si les textes, à notre connaissance, livrent peu de renseignements sur le sujet, il semble difficile que les épures réalisées, par exemple, au XIII ${ }^{\mathrm{e}}$ siècle à la cathédrale de Soissons, avec leur longueur de plus de 
sept mètres, aient été réalisées par un homme seul. Et les comptes de construction de la cathédrale de Troyes paraissent le confirmer puisqu'en 1381-1382, c'est à Michelin et Jean Tierri que sont versés vingt sous "pour faire une aire en la ramure [dans les combles] de costé le gros clochier pour faire les portrais dou jubé en la $2^{\mathrm{e}}$ sepmaine de juillet » et cinq sous « pour faire le pourtrait dou jubé en une pel de parchemin $»^{17}$.

La solitude de l'architecte médiéval semble bien, au moins dans certains cas, à nuancer. Les qualificatifs d'exception eux-mêmes se partagent parfois, si l'on en juge au pluriel rencontré dans la mention d'un accord passé, en 1271, pour la cathédrale de Trogir : cum professoribus lapicidis (avec des professeurs tailleurs de pierre) ${ }^{18}$. Mais ces collaborations, ces échanges sont-ils uniquement ponctuels ou s'inscrivent-ils dans une durée?

\section{Une équipe dans l'ombre de l'architecte}

13 La difficulté que l'on peut avoir à saisir l'identité ou le rôle des dépendants entourant le chef d'entreprise médiéval ne se limite en rien au domaine de la construction et rejoint les problèmes rencontrés plus largement dans l'appréhension de la famille elle-même. Les deux (famille et entreprise) se confondent souvent, du reste, dans la mesure où, comme le rappelle Didier Lett, la famille " est aussi une unité économique regroupant un ensemble de producteurs et de consommateurs $»^{19}$. Il n'est en effet pas rare de rencontrer des dynasties de maîtres d'œuvre ou d'architectes du type de celle des Chambiges $^{20}$. Le débat engagé sur la cathédrale de Gérone en 1416 fait, lui-même, intervenir Pasqual de Julbe, maître majeur de la cathédrale de Tortosa et Joan de Julbe, «son fils et collaborateur $~^{21}$. Comment imaginer, surtout dans le cas de personnages aussi éminents, que le fils n'ait pas été associé à l'élaboration des projets portés par le père ? Outre les fils, ce sont aussi les frères que l'on trouve engagés dans diverses entreprises communes de construction. Le maître d'œuvre de la cathédrale de Carpentras, Blaise L'Écuyer arrive ainsi dans la capitale du Comtat avec ses deux frères, Jean et Pierre, et se voit remplacé à ce poste par les Marseillais Pierre et Jean Blachière ${ }^{22}$.

Que dire, de même, du rôle respectif des associés que l'on retrouve à la tête de nombreux chantiers médiévaux et dont certains contrats précisent qu'ils devront s'enseigner mutuellement ce qu'ils savent de leur art ${ }^{23}$ ? L'association n'est en effet pas réservée aux petits artisans manquant de moyens. Hélion L'Auvergnat, qui deviendra maitre des œuvres de maçonnerie du comte de Provence et prit, entre autres, en charge la construction de la dernière travée de la cathédrale d'Aix-en-Provence, en 1472, y recourt par exemple quelques années plus tôt quand, en 1464, il bâtit avec son collègue Pierre Mauron, lapicide, une chapelle dans cette même église ${ }^{24}$.

Le maître peut ainsi se retrouver à la tête d'un groupe domestique large, pour partie impliqué dans ses affaires. Les registres d'imposition particulièrement précis de Trévise, étudiés par Matthieu Scherman, permettent ainsi de constater que Lorenzo da Quinto, le plus important maçon de la ville, fils et père de maçons, est à la tête d'une «famille» de quinze personnes qui comprend entre autres son frère, également maçon ${ }^{25}$. Pour nombre de chantiers, de la fin du Moyen Âge, la pratique de la prise des travaux à forfait place les exécutants dans l'ombre du chef d'entreprise qui est seul à signer le contrat. Cette manière de procéder était répandue dans l'architecture courante mais elle se retrouve également dans les chantiers les plus importants comme 
celui, par exemple, du Palais des Papes d'Avignon, au XIV siècle. Jean de Louvres, qui fut le maitre d'œuvre du palais sous Clément VI (1342-1352) et Innocent VI (1352-1362) passa plusieurs de ces conventions avec la Chambre apostolique. Ce n'est qu'au détour de rares mentions comptables que nous découvrons qu'il travaille à Avignon avec son cousin Harrouet Godefridi et confie la responsabilité du chantier, quand il s'absente, à un certain Guillaume Richome, désigné comme preparator et director operis (préparateur, directeur de l'œuvre ${ }^{26}$. Ce dernier peut être assimilé à ce que l'on désigne sur les chantiers comme un appareilleur: "Chef ouvrier qui, sachant l'art de la coupe des pierres, les trace de la grandeur et de la figure qu'elles doivent avoir, suivant la place où il les destine, et qui en outre dirige le travail des tailleurs de pierre, des poseurs et contreposeurs $"{ }^{27}$. Ce personnage assiste le maitre d'œuvre, il est un intermédiaire entre ce dernier et les ouvriers et assume une partie du travail de dessin et/ou de traçage ${ }^{28}$. Il s'agit d'un rouage important pour la mise en œuvre du projet sur les gros chantiers et un membre essentiel de la direction technique des travaux. C'est lui qui permet à l'architecte de ne pas être constamment présent sur ses chantiers en prenant en charge une partie de la planification et de la préparation du travail de construction. Ainsi l'architecte n'apparaît-il pas, quand les sources sont assez précises pour permettre de le saisir, comme un homme isolé mais comme le chef d'équipe ou d'une familia relativement complexe et, on ne peut que le regretter, difficile à saisir.

Experts, parents, associés, assistants..., les sources médiévales mettent en scène de manière fugace divers personnages, uniquement masculins, de l'entourage professionnel de l'architecte qui peuvent être amenés à intervenir à divers niveaux dans l'élaboration des projets. Sans doute faudrait-il y ajouter les personnes à même de tenir les comptes, de négocier l'achat des matériaux et de recruter la main d'œuvre dans la mesure où l'architecte se présente souvent comme un véritable entrepreneur. La plupart de ces figures de second plan (pour nos sources) demeure à tout jamais dans l'ombre des grands noms qui seuls sont à même de prendre la lumière. Peut-on qualifier les équipes qu'ils constituent d'embryons d'agences d'architecture ? Il est bien difficile de trancher en l'état de nos connaissances dans le domaine. Des pistes de recherche s'ouvrent néanmoins qui, à terme, pourront peut-être permettre de préciser les rôles et de replacer l'architecte ou le maître d'œuvre médiéval dans un environnement de travail à dimension plus humaine qu'héroïque.

\section{NOTES}

1. Nikolaus Pevsner, "The Term 'Architect' in the Middle Ages ", Speculum 17, no. 4, Cambridge (Massachusetts), The mediaeval academy of America, 1942, p.549-562. Voir aussi le bilan plus récent établi par Louis Callebat, " "Architecte" : histoire d'un mot ", Voces, 10-11, 1999-2000, Salamanque, Universidad de Salamanca, p. 47-58.

2. Journal Officiel, Arrêté du 17/02/1986 - date de la publication : 22/09/2000 - éd. commission de l'équipement et des transports.

3. Ce titre est emprunté au travail majeur que Patrick Boucheron a consacré à cette question : Patrick Boucheron, «L'architecte comme auteur. Théorie et pratique de la création 
architecturale dans l'Italie du Quattrocento ", Michel Zimmermann (dir.), Auctor \& auctoritas. Invention et conformisme dans l'écriture médiévale, Paris, École des Chartes, 2001, p. 531-552.

4. Peter Cornelius Claussen, «Kathedralgotik und Anonimtät, 1130-1250 ", Wiener Jahrbuch fur Kunstgeschichte, t. 46-47, 1993-1994, p. 141-160.

5. Patrick Boucheron, « L'architecte... », op. cit., p. 536.

6. Ibid., p. 540.

7. Franco Borsi, Bramante, trad.Fr., Milan-Paris, Electa, 1990, 357 p., p. 34, cité par Patrick Boucheron, «L'architecte... ", op. cit., p. 533.

8. Voir sur ces personnages, les notices qui leur sont consacrées par Isabelle Isnard dans Pascale Charron et Jean-Marie Guillouët (dir.), Dictionnaire d'histoire de l'art du Moyen Âge occidental, Paris, Robert Laffont, 2009, 1131 p.

9. Citons simplement les travaux de Pierre du Colombier, Les chantiers des cathédrales, Paris, Picard, [1953] 1973, 189 p., ou Marcel Aubert, "La construction au Moyen Âge ", Bulletin monumental, t. 118 (1960), p. 241-259, et t. 119 (1961), p. 7-42, 81-120, 181-209, 297-323.

10. Texte édité dans Victor Mortet et Paul Deschamps, Recueil de textes relatifs à l'histoire de l'architecture et à la condition des architectes en France au Moyen Âge, Paris, CTHS, [1911 (t. I), 1929 (t. II)], réed. 1995, 1100 p., p. 927.

11. La littérature sur ces débats est trop abondante pour envisager d'en donner ici une bibliographie exhaustive. Voir notamment James S. Ackerman, « "Ars sine scientia nihil est" Gothic Theory of Architecture at the Cathedral of Milan», The Art Bulletin, t. 31, 1949, p. 84-111; Christian Freigang, «Solemnius, notabilius et proporcionabilius. Les expertises de la construction de la cathédrale de Gérone. Réflexions sur le discours architectural au Moyen Âge ", dans Fabienne Joubert et Dany Sandron, Pierre, lumière, couleur. Études d'histoire de l'art du Moyen Âge en l'honneur d'Anne Prache, Paris, Presses de l'Université Paris-Sorbonne, 1999 (Cultures et civilisations médiévales-XX), p. 385-393 ; Sandrine Victor, « L'expertise architecturale au service d'une décision controversée : la cathédrale de Gérone, 1386 et 1416 ", dans Experts et expertise au Moyen Âge. Consilium quaeritur a perito, XLII ${ }^{\mathrm{e}}$ Congrès de la SHMESP, Paris, Publications de la Sorbonne, 2012, p. 243-253 ; Joan Domenge i Mesquida et Jacobo Vidal Franquet (dir.), Visurar l'arquitectura gótica : inspeccions, consells i reunions de mestres d'obra (s. XIV-XVIII), Palerme, Caracol, 2017, 339 p.

12. James S. Ackerman, " "Ars sine scientia... ", op. cit.

13. Voir Experts et expertise au Moyen Âge. Consilium quaeritur a perito, XLII Congrès de la SHMESP, Paris, Publications de la Sorbonne, 2012, 355 p. ; Claude Denjean et Laurent Feller (dir.), Expertise et valeur des choses I. Le besoin d'expertise, Madrid, Casa de Velázquez, 2013, 279 p.; Laurent Feller et Ana Rodríguez (dir.), Expertise et valeur des choses II Savoirs, écriture, pratiques, Madrid, Casa de Velázquez, 2016, 471 p.

14. Voir notamment Fanny Madeline, «Formes et figures de l'expertise dans les chantiers royaux en Angleterre aux XII ${ }^{\mathrm{e}}$ et XIII ${ }^{\mathrm{e}}$ siècles : des compétences techniques au service du politique», dans Experts et expertise... op. cit., p. 228-242 ; Marie-Anne Moulin, «Experts et expertise sur les chantiers de construction en Normandie méridionale: l'exemple de la ville d'Argentan au $\mathrm{XV}^{\mathrm{e}}$ siècle ", ibidem, p. 255-267 ; Philippe Bernardi, « Du maître expert à l'expert. Réflexions sur l'évolution de la référence à l'expertise dans le bâtiment, entre XIV et XVI siècle ", dans Claude Denjean et Laurent Feller (dir.), Expertise et valeur des choses au Moyen Âge. I. op. cit., p. 109-129.

15. Voir Fanny Madeline, op. cit. Pierre-Yves Le Pogam, Les maîtres d'œuvre au service de la papauté dans la seconde moitié du XIII siècle, Rome, École française de Rome, 2004 (Collection de l'École française de Rome, 337), mais également les diverses contributions consacrées à ces maîtres des œuvres dans le domaine français dans Odette Chapelot (dir.), Du projet au chantier. Maîtres d'ouvrage et maîtres d'œuvre aux XIV ${ }^{e}-X V^{e}$ s., Paris, EHESS, 2001, par exemple.

16. Texte édité dans Victor Mortet et Paul Deschamps, Recueil de textes... op. cit., p. 873.

17. Stephen Murray, Building Troyes Cathedral, the Late Gothic campaigns, BloomingtonIndianapolis, Indiana University Press, 1987, 257 p. 
18. Marina Šimunić Buršić, "La voûte gothique de type angevin en Croatie », dans François Fleury, Laurent Baridon, Antonella Mastrorilli, Rémy Mouterde et Nicolas Reveyron (dir.), Les temps de la construction. Processus, acteurs, matériaux, Paris, Picard, 2016, p. 505-515 (p. 507).

19. Didier Lett, Famille et parenté dans l'Occident médiéval $V^{e}-X V^{e}$ siècle, Paris, Hachette, 2005, 255 p., p. 144.

20. Florian Meunier, Martin et Pierre Chambiges. Architectes des cathédrales flamboyantes, Paris, Picard, 2015, $359 \mathrm{p}$.

21. Sandrine Victor, « L'expertise architecturale... », op. cit., p. 247.

22. Alain Girard, L'Aventure gothique entre Pont-Saint-Esprit et Avignon du XIII ${ }^{e}$ au XV siècle, Aix-enProvence, Edisud, 1996, 190 p., p. 149-152.

23. Philippe Bernardi, Métiers du bâtiment et techniques de construction à Aix-en-Provence à la fin de l'époque gothique (1400-1550), Aix-en-Provence, Publications de l'Université de Provence, 1995, 501 p., p. 78.

24. Voir Philippe Bernardi, «Entre mobilités sociale et géographique : les pérégrinations d'un tailleur de pierre français dans la Provence du XVe siècle ", dans Jacques Dubois, Jean-Marie Guillouët et Benoît Van den Bossche (dir.), Les transferts artistiques dans l'Europe gothique, Paris, Picard, 2014, 367 p., p. 177-188.

25. Matthieu Scherman, Familles et travail à Trévise à la fin du Moyen Âge, Rome, École française de Rome, 2013, 684 p., p. 223.

26. Philippe Bernardi, Philippe Dautrey et Jean-Marc Mignon, «Jean de Louvres : un maître des œuvres du Palais des Papes d'Avignon (1342-1358)», dans Chapelot O. (dir.), Du projet au chantier..., op. cit. , p. 37-58.

27. Sophie Jossier, Dictionnaire des ouvriers du bâtiment, Paris, Librairie générale de l'architecture et des travaux publics, 1881,450 p., p 8.

28. Voir les divers exemples qu'en donne Marcel Aubert, « La construction... », op. cit., p. 11.

\section{RÉSUMÉS}

Si la notion d'« agence d'architecture » peut paraitre anachronique, s'agissant de construction médiévale, elle a le mérite d'interroger l'historien sur le caractère collectif de l'élaboration des projets. Alors que la figure éminente de l'architecte semble écraser toute perspective de travail en équipe, nous verrons que l'analyse des sources médiévales permet de nuancer cette vision hiératique. Quelques situations d'échanges se font jour, quelques collaborations s'entrevoient, quelques partages de responsabilités apparaissent. La documentation conservée, dans ce domaine comme dans d'autres, occulte en grande partie les personnages de second plan au profit des chefs d'entreprise ou de famille. Ce n'est que par touches, en sollicitant une documentation concernant tout à la fois les trois derniers siècles du Moyen Âge et plusieurs pays d'Occident, que quelques éléments de réflexions peuvent être avancés sur le sujet. Cette première approche ne peut prétendre répondre de manière catégorique aux questions posées. Elle ouvre des pistes qui, à terme, pourront peut-être permettre de préciser les rôles et de replacer l'architecte ou le maître d'œuvre médiéval dans un environnement de travail à dimension plus humaine qu'héroïque.

Although the notion of "architectural firm" may seem anachronistic in the context of medieval construction, it has the merit of questioning the historian about the collective nature of project 
development. While the eminent figure of the architect seems to crush any prospect of teamwork, we shall see that the analysis of medieval sources allows us to qualify this hieratic vision. Some situations of exchange emerge, some collaborations are glimpsed, and some sharing of responsibilities occurs. The documentation that has been preserved, in this field as in others, largely conceals the secondary characters in favour of the heads of companies or families. It is only in small steps, by soliciting documentation concerning the last three centuries of the Middle Ages and several Western countries, that some elements of reflection can be put forward on the subject. This first approach cannot claim to provide categorical answers to the questions posed. It opens up avenues which, in the long term, may make it possible to clarify the roles and place the medieval architect or master builder in a working environment with a human rather than heroic dimension.

Auch wenn der Begriff des "Architekturbüros" auf den ersten Blick anachronistisch erscheinen mag, wenn es um mittelalterliche Bauten geht, so kann er im Sinne einer historiographischen Forschung doch dazu dienen, nach dem kollektiven Charakter der Ausarbeitung von Bauprojekten zu fragen. Während die scheinbar herausragende Figur des Architekten oft die Perspektive auf eine Gemeinschaftsarbeit überstrahlt, so erlaubt die genauere Untersuchung mittelalterlicher Quellen es doch, diese hierarchische Sicht zu differenzieren. So erscheinen dabei Hinweise auf Austausch, Zusammenarbeit oder auch geteilte Verantwortung. Wie auch in anderen Bereichen erlaubt es die Überlieferung kaum, die im Hintergrund hinter einem Geschäftsleiter oder Familienoberhaupt tätigen Personen zu benennen. Nur durch vereinzelte Quellen und die Recherche über drei Jahrhunderte in mehreren westlichen Ländern hinweg lassen sich einige Aussagen zu diesem Thema treffen. Die in diesem Beitrag vorgenommene erste Annäherung kann nur wenige Antworten auf die gestellten Fragen liefern; sie öffnet jedoch weitere Forschungshorizonte, um es zukünftigen Recherchen zu erlauben, die jeweiligen Rollen der beteiligten Akteure genauer zu definieren und die Figur des mittelalterlichen Architekten oder Baumeisters in seinem Arbeitsumfeld zu kontextualisieren und aus einer heldenhaften in eine vielmehr menschliche Dimension zu rücken.

\section{INDEX}

Mots-clés : Moyen Âge, architecte, projet, maître d'œuvre, expertise

\section{AUTEUR}

\section{PHILIPPE BERNARDI}

Philippe Bernardi, directeur de recherche au CNRS (LaMOP, CNRS-Université Paris 1 PanthéonSorbonne), est médiéviste et spécialiste du monde de la construction. Ses travaux portent principalement sur l'histoire économique et sociale des techniques dans le Midi méditerranéen, envisagée principalement à partir des sources écrites. Il a écrit et dirigé plusieurs ouvrages sur ces questions dont Bâtir au Moyen Âge (Paris, éd. du CNRS, 2011), Maître, valet et apprenti au Moyen Âge. Essai sur une production bien ordonnée (Toulouse, CNRS-Université Toulouse-Le Mirail, 2009). Il a notamment dirigé, avec Patrice Beck et Laurent Feller, Rémunérer le travail au Moyen Âge. Pour une histoire sociale du salariat (Paris, Picard, 2014) et, avec Corine Maitte et François Rivière, Dans les règles du métier. Les acteurs des normes professionnelles au Moyen Âge et à l'époque moderne (Palerme, New Digital Frontiers, 2020). Adresse électronique :

Philippe.Bernardi@univ-paris1.fr 